\title{
DO SONGBIRDS PERCEIVE SYLLABLES AS ISOLATED TOKENS?
}

\author{
TOMOKO MIZUHARA $^{1}$ and KAZUO OKANOYA ${ }^{* 1}$ \\ *Corresponding Author: cokanoya@mail.ecc.u-tokyo.ac.jp \\ ${ }^{1}$ Department of Life Sciences, The University of Tokyo, Tokyo, Japan
}

The songbird is an established model for comparative biology of human speech acquisition (Marler, 1970; Doupe \& Kuhl, 1999). Many attempts to explore further parallels have been made to date: some described song syntax of Bengalese finches (e.g. Okanoya, 2004) and others trained zebra finches in artificial grammar composed of syllables (e.g. van Heijningen et al., 2013). Here they share a tacit assumption — songbirds consider syllables as tokens.

It is a common practice for researchers in this field to isolate each syllable from birdsong for analysis. However, songbirds basically do not vocalize individual syllables apart from song bouts. In addition, Dooling \& Searcy (1980) pointed out that auditory masking effects may not be ignorable when song notes are perceived.

We hypothesized that if songbirds perceived syllables as tokens, they would detect a syllable regardless of temporal distance from another syllable. 6 female Bengalese finches were first trained to detect syllable [a] that were coupled with another syllable [p]. In p-t condition [p] preceded [a]; in $t-p$ condition $[p]$ followed [a]. The interval had 4 variants that were uncommon in Bengalese finch song (Figure 1A). After reaching a predetermined pass criterion, probe stimuli were introduced. They had shorter or longer interval than that of training stimuli (Figure 1A).

Birds with odd ID went through p-t condition first, and birds with even ID went the other way around (Figure 1B). Difference in mean days each group took for the first condition was not significant (Welch's two sample ttest, $t=4.05, d f=2, P=0.055)$. Once they finished either condition, they did not require so long days to get accustomed to new conditions (paired ttest, $t=5.87, d f=5, P=0.0020$ ). 
Figure 1C shows that response rate decreased as interval was shortened in $\mathrm{p}$-t condition except bird\#4, but not in t-p condition. We fitted data of each condition with generalized linear mixed models (GLMMs). The null model (the effect of $\log$ (gap length) equals 0$)$ was rejected in p-t condition $(P$ $<0.001)$ but not in t-p condition $(P=0.47)$ with likelihood ratio test. This result is consistent with the fact that effect of forward masking is greater than that of backward masking (Dooling \& Searcy, 1980). In other words, tokenization was inhibited in $\mathrm{p}$-t condition in spite of the training with variable intervals.

Overall, it is not taken for granted that songbirds regard syllables as tokens unconditionally. We do not think that the brief interval itself was distracting for birds: If so, their response rate would have shown some decline in t-p condition, too. Response rate in t-p condition, however, kept high independent of the stimulus type. Moreover, in $\mathrm{p}$ - $t$ condition, the longer the interval was, the higher the response rate was. These raises the possibility that tokenization might be helped by elongating inter-syllable interval. Such speculation is also consistent with the result of training days comparison between before and after switching, as birds, especially with odd ID (p-t first), soon passed their novel condition. We hope that our study will benefit future artificial grammar learning studies not only in songbirds, but also in other animals including humans.
A

B
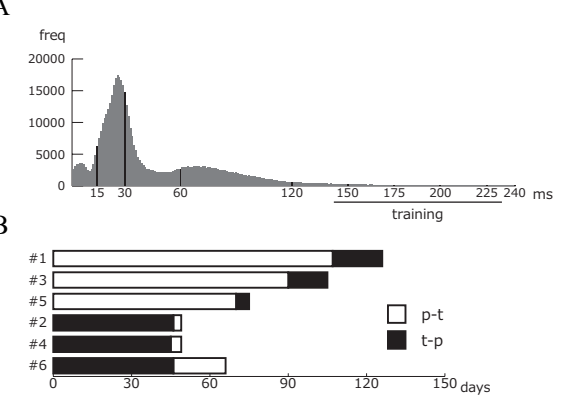

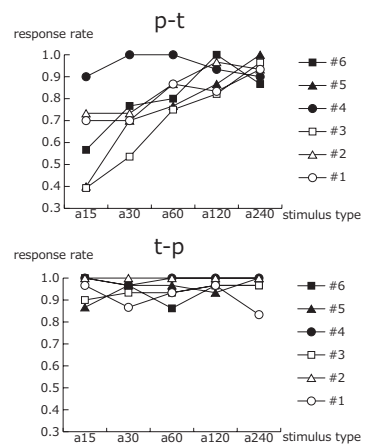

Figure 1 (A) Distribution of gap length in Bengalese finch song (data from 8 males). Numbers below represent length of silent intervals adopted in this study. A black bar indicates 4 variants for training stimuli. (B) Training days required for discrimination. (C) Response rate to probe stimuli.

\section{Acknowledgements}

We thank Dr. Gabriël J. L. Beckers for his comments on the experimental plan, Dr. Takuya Koumura for gap distribution data of Bengalese finch song, Ms. Tomoko Fujii for providing song data to make stimuli, and Ms. Yoshiko Sawada for her cooperation in bird care. This study was supported by MEXT Innovative Areas \#4903, JP17H06380 to K.O. and by the University of Tokyo Grant for PhD Research RA to T.M.. 


\section{References}

Dooling, R. J., \& Searcy, M. H. (1980). Forward and backward auditory masking in the parakeet (Melopsittacus undulatus). Hearing research, 3(4), 279-284.

Doupe, A. J., \& Kuhl, P. K. (1999). Birdsong and human speech: common themes and mechanisms. Annual review of neuroscience, 22(1), 567-631.

Marler, P. (1970). Birdsong and Speech Development: Could There Be Parallels? There may be basic rules governing vocal learning to which many species conform, including man. American Scientist, 58(6), 669-673.

Okanoya, K. (2004). Song syntax in Bengalese finches: proximate and ultimate analyses. Advances in the Study of Behavior, 34, 297-346.

van Heijningen, C. A., Chen, J., van Laatum, I., van der Hulst, B., \& ten Cate, C. (2013). Rule learning by zebra finches in an artificial grammar learning task: which rule? Animal cognition, 16(2), 165-175. 